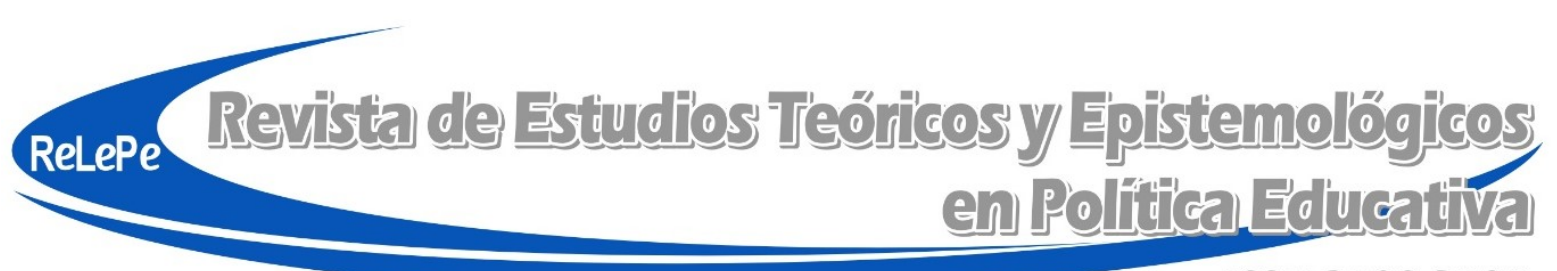

ISSN 2409-3696

DOI: $10.5212 /$ retepe.v.4.021

\title{
El planeamiento educativo: función política, modelos y algunas experiencias latinoamericanas
}

\author{
Nicolás Bentancur \\ Universidad de la República (Uruguay) \\ nicobent63@gmail.com \\ ORCID: https://orcid.org/0000-0003-4909-6712
}

Resumen: La función de planeamiento en el marco de la gestión pública está asociada a determinantes más comprehensivas que aluden a la extensión de las funciones del Estado y a las distintas modalidades de construcción de las políticas públicas. Partiendo de ese supuesto, en el texto se pasa revista a su traducción al campo de las políticas de enseñanza, haciendo referencia a los orígenes y evolución de la planificación educativa y a las condiciones particulares de ese proceso en América Latina. Asimismo, se presentan distintos modelos de planeamiento, y se discute su adecuación a las necesidades de las políticas educativas de la región en la coyuntura actual.

Palabras-clave: Planeamiento Educativo. Políticas Educativas. América Latina.

\section{Planejamento educacional: função política, modelos e experiências latino americanas}

Resumo: A função de planejamento no âmbito da gestão pública está associada a determinantes mais abrangentes que se referem à extensão das funções do Estado e às diferentes modalidades de construção de políticas públicas. Com base nessa premissa, o texto revisa sua tradução para o campo das políticas educacionais, referindo-se às origens e evolução do planejamento educacional e às condições particulares desse processo na América Latina. Da mesma forma, são apresentados diferentes modelos de planejamento e discutida sua adaptação às necessidades das políticas educacionais da região no estágio atual.

Palavras-chave: Planejamento Educacional. Políticas Educacionais. América Latina.

\section{Educational planning: political function, models and Latin American experiences}

\begin{abstract}
The planning function within the framework of public management is associated with more comprehensive determinants that refer to the extension of the functions of the State and the different modalities of construction of public policies. Based on this assumption, the text reviews its translation to the field of education policies, referring to the origins and evolution of educational planning and the particular conditions of that process in Latin America. Likewise, different planning models are presented, and their adaptation to the needs of the educational policies of the region in the current situation is discussed.
\end{abstract}

Keywords: Educational Planning. Educational Policies. Latin America. 


\section{Introducción}

En este texto nos proponemos encuadrar conceptualmente a las actividades de planeamiento educativo -entendidas en un nivel macro- en tanto instrumento y estrategia para las políticas públicas de educación, con especial referencia a América Latina.

Con ese objetivo, en primer término abordaremos la cuestión más amplia del relacionamiento entre los fines del Estado, las políticas públicas y los sistemas y actividades de planificación. Estas primeras definiciones son necesarias por cuanto el planeamiento que se desarrolla en el ámbito público se inscribe en determinantes más generales, definidas por el rol asignado al Estado en una sociedad determinada, y por el tipo de estrategia de construcción de las políticas públicas preferido para propiciar las transformaciones que se consideren deseables. Esto es, por la naturaleza en última instancia política de las funciones de planeación.

Luego nos abocaremos específicamente a la planificación educativa, reparando en su surgimiento y evolución y en las especificidades del campo, que se vinculan a la elucidación de los formatos y modalidades más eficaces disponibles para informar apropiadamente las políticas del sector.

Posteriormente, presentaremos un rápido recorrido por las experiencias y desarrollos de la planificación educativa en la región, procurando destacar las innovaciones y jalones más importantes de esa evolución.

Para finalizar, propondremos unas breves reflexiones sobre la oportunidad del empleo de ciertos modelos y estrategias disponibles para organizar el planeamiento de la educación, en el contexto histórico actual de las políticas educativas de la región.

\section{El vínculo entre fines del Estado y planificación estatal}

La determinación del concepto y el rol de la planificación pública en una esfera colectiva determinada -en nuestro caso, las políticas educativas- no puede establecerse cabalmente sin una remisión a la cuestión más general de los territorios reservados para el Estado y para el mercado en cada sociedad. En el devenir histórico los cometidos estatales han variado sustantivamente, de época en época y de país en país, desde esquemas absolutistas en un extremo a armados liberales en otro, en un movimiento de flujos y reflujos sucesivos que explicitan la naturaleza polémica y cambiante de este clivaje público - privado vertebrador del ordenamiento social.

Si acotamos temporalmente el radio de análisis, podemos identificar desde finales del siglo XIX, y con particular énfasis desde el segundo período de posguerra del siglo XX, el proceso de constitución y desarrollo de un modelo de relacionamiento público - privado caracterizado por una activa participación del Estado en la economía y por el suministro público de una pluralidad de bienes y servicios sociales, que fue denominado welfare state o Estado de Bienestar. La novedad se corresponde con el reconocimiento de la tercera dimensión de la ciudadanía -la económica-, que sucedió a sus facetas civil en el siglo XVIII, y política en el XIX. El Estado de Bienestar constituiría, entonces, la culminación de un proceso de expansión progresiva de los derechos ciudadanos (MARSHALL, 1965).

Siguiendo a Mishra (1989, p. 56), por Estado de Bienestar entendemos a un sistema social caracterizado por:

a) intervención estatal en la economía para mantener el pleno empleo o, al menos, garantizar un alto nivel de ocupación; 
b) provisión pública de una serie de servicios sociales, tales como educación, asistencia sanitaria, pensiones, ayudas familiares y vivienda, caracterizada por su universalidad, esto es, su direccionamiento hacia todos los grupos de renta de la población, con independencia del nivel de sus ingresos; $y$

c) responsabilidad estatal en el mantenimiento de un nivel mínimo de vida, con categoría de derecho social y no de caridad.

El cumplimiento de cometidos estatales de tal amplitud, complejidad y diversidad exigía la movilización de recursos humanos y materiales de acuerdo a criterios racionales, afincados en un conocimiento certero de la realidad, en objetivos y metas claramente definidas y en procedimientos hábiles para procesar el tránsito desde un punto hasta otro. En otros términos, requería un plan. Y como se ha sostenido, la idea de plan presupone tres axiomas: a) que el saber no sólo permite conocer, sino también manejar/dirigir la realidad; b) que la razón es capaz de discernir qué es lo mejor en una situación determinada; y c) que la voluntad tiene la potencialidad necesaria para dirigir los procesos sociales (AGUERRONDO, 2007, p. 3-4).

No es casual que la planificación moderna se haya desarrollado históricamente en coyunturas históricas y geográficas en las que los estados nacionales expandían sus funciones: como antecedentes, en la Rusia posrevolucionaria y en el Estados Unidos del "New Deal", y más extendidamente, en Europa y Japón luego de la segunda guerra mundial, y de manera coetánea aunque recortada- en América Latina. Bajo formatos de gobierno disímiles (socialistas, capitalistas de economía mixta, socialdemócratas o populistas), el "plan" orientó la ejecución de las nuevas misiones económicas y sociales de los estados, constituyéndose en ocasiones en una previsión y orientación exhaustiva de la evolución de las más diversas facetas de la vida social (desde la economía hasta el desarrollo urbano y territorial, comprendiendo entre otras también a la educación). Se trata en todos los casos de la asunción de perspectivas desarrollistas, esto es, de la convicción de que el pasaje de las naciones a estadios superiores de prosperidad económica e integración social depende de la orientación y del empuje de los estados.

La planificación ha sido interpelada -con creciente predicamento a partir de la década de 1980- desde visiones pluralistas liberales, que cuestionaron tanto su aptitud para conseguir los fines planteados, como la afectación de espacios de determinación que deberían ser delegados a los mercados y los individuos. El planteo de Aguilar Villanueva (1992, p. 26-27) da cuenta cabal de estas posturas:

[...] gobernar de acuerdo a plan exige grandes dosis de control autoritario, o bien masivo consenso y apoyo político [...] el plan, incluido el proceso de planeación, requiere un consenso tan global e intenso que las democracias plurales no les pueden dar [...] el pluralismo político, las libertades económicas $\mathrm{y}$ las perspectivas culturales diferentes terminan por volver imposible la gigantesca capacidad de computación y de consenso que el gobierno necesita para gobernar de acuerdo al plan nacional global [...] la acción social desborda la planificación gubernamental [...].

Es evidente que en este punto la contraposición alude a premisas filosóficas. Como se preguntaba Bertrand Russell, “¿cómo podemos combinar el grado de iniciativa individual necesario para el progreso, con el grado de cohesión social indispensable para sobrevivir?” (RUSSEL, 1995, p. 9).

Estas críticas se han alineado con la alteración del supuesto fáctico que, como señaláramos, ambientó el surgimiento de la planificación: la extensión de los estados de bienestar, y su sustitución por formatos más acotados de intervención estatal en la economía en la sociedad. 
Naturalmente, modelos de desarrollo basados en el protagonismo de las fuerzas del mercado no requieren -ni se compadecen- con un "plan" decidido políticamente, sino que más bien tienden a asociarse con el impulso de "marcos de políticas" que fijan solamente algunas reglas generales que establecen límites básicos a su accionar, o a lo sumo constituyen sistemas de incentivos para la promoción de determinadas conductas.

Según estas miradas escépticas sobre la posibilidad y el mérito de los ensayos de planeación, "gobernar por planes" conlleva una serie de complejidades de difícil resolución. Por un lado, exige congregar las conductas de todos los actores sociales mediante formas de control autoritario, o alternativamente, de la existencia de un consenso y apoyo político generalizado, difícilmente obtenible en el marco de sociedades plurales y heterogéneas, cuyo desenvolvimiento desborda los objetivos e instrumentos que un gobierno considera preferibles. Por otra parte, por sus pretensiones omnicomprensivas requiere de grandes capacidades de anticipación y cálculo acerca de la evolución de los problemas y la validez de las soluciones, careciendo de la flexibilidad necesaria para adaptarse a situaciones cambiantes. En cambio, el "gobierno por políticas" se compadecería mejor con un accionar público más limitado y flexible, con el desarrollo de modelos y prácticas diversas y con un mayor protagonismo de los actores sociales.

Empero, se advierte que también las fórmulas más acotadas de "gobierno por políticas" tienen sus carencias. La ausencia de un plan director conspira contra la debida articulación entre diferentes programas, induce el accionar de la administración hacia prácticas meramente responsivas y cortoplacistas, y tiende a separar rígidamente la regulación de un sector social entre actividades de dirección, resueltas en el centro del sistema de acuerdo a modalidades comúnmente tecnocráticas, y a prácticas efectivas libradas a la sociedad por fuera del andamiaje de autoridad. Asimismo, suelen ser ineficaces para enfrentar problemas complejos y arraigados, que requieren de lapsos prolongados de intervención pública sostenida y coherente para arribar a soluciones satisfactorias.

Por cierto, el instrumento de la planeación no es de por sí una respuesta necesaria y suficiente a cada uno de estos desafíos, y la frustración de experiencias de esa naturaleza que tuvieron su auge cinco décadas atrás obliga a su ponderación crítica. Mas resulta conveniente distinguir analíticamente dos tipos de cuestionamientos que se han lanzado sobre el planeamiento: uno, vinculado a determinadas concepciones sobre el rol deseable del Estado y de los gobiernos en la conducción social. Otro, claramente diferenciable, que puso en tela de juicio el clásico modelo normativo de planeación, de carácter verticalista y cuño tecnocrático.

Para el primer cuestionamiento, cualquier intento de planeación que vaya más allá de un "plan marco" es política y filosóficamente indeseable, por cuanto expande indebidamente el rol del estado y afecta la libertad de individuos para tomar sus propias decisiones. Por ende, quienes suscriban estas premisas se mostrarán adversos a cualquier forma de ensayo ambicioso de planeamiento.

En cambio, para los críticos del modelo tradicional la planificación debe reformularse en torno a un armado más amplio y plural, que defina por expedientes democráticos los objetivos sociales, y aplique su instrumentación operativa (lineamientos, medidas) de manera flexible y teniendo en cuenta los imperativos situacionales. Se manifestarán partidarios, entonces, de estrategias que involucren a múltiples actores en una construcción educativa de carácter comunitario. Como puede advertirse, no hay en esta segunda versión una impugnación a las finalidades políticas del planeamiento.

Sobre la traducción de estas críticas a la experiencia de planificación de América Latina y el surgimiento de estrategias alternativas volveremos más abajo. Pero antes se hace necesario 
precisar qué entendemos por planificación educativa, y esbozar los jalones principales de su evolución histórica.

\section{La planificación educativa: concepto, surgimiento y evolución}

Se parte aquí de un concepto amplio del planeamiento educativo, entendido como el proceso a través del cual los problemas son analizados y las políticas son generadas, implementadas, evaluadas y rediseñadas (HADDAD; DEMSKY, 1995).

En la medida en que las actividades de planeamiento forman parte de las estrategias de intervención estatal para orientar las políticas públicas, las prioridades y las elecciones individuales en una dirección considerada deseable para el progreso social, el planeamiento específicamente educativo se basa en dos supuestos. El primero es de índole finalista: dado el valor social de la educación, sus objetivos pueden ser mejor resueltos cuando las decisiones se adoptan en espacios colectivos y no exclusivamente individuales. Por lo tanto, el Estado ejerce así su poder y autoridad para diseñar y llevar a cabo las políticas educativas dirigidas a los ciudadanos. El segundo supuesto que fundamenta las actividades de planificación es de naturaleza instrumental: en la medida en que el sector educativo consume una porción importante de los recursos públicos, es menester que estos sean asignados adecuadamente y utilizados de manera eficiente (VARGHESE, 2011).

Para llegar a esas definiciones, el planeamiento educativo recorrió un largo periplo histórico. ${ }^{1}$ Durante varias décadas y hasta la Segunda Guerra Mundial adoleció de serios vicios: cortoplacismo (proyecciones a un año o poco más), fragmentación en la cobertura de los distintos niveles del sistema de enseñanza, desarticulación con otras políticas que atendían a necesidades sociales y económicas, e inmovilismo para adaptarse a situaciones cambiantes. Como en otras múltiples manifestaciones del desarrollo humano, también en esta esfera el gran conflicto bélico generó poderosos incentivos para la transformación de las prácticas antiguas y el cambio institucional. Pero esa nueva etapa tuvo características distintas según el grado de desarrollo económico de los países.

En los países industrializados, entre los años 1945 y 1970 se transitó por cuatro fases sucesivas: la reconstrucción, la escasez de mano de obra, la expansión rampante y la innovación. Es en esta última fase, de finales de los años sesenta, en que los sistemas educativos tradicionales son desafiados por los profundos cambios sociales, económicos y en el conocimiento mismo. Esta contradicción da lugar a la llamada "Gran Explosión de la Educación" en el año 1967, marcado por protestas estudiantiles que recibieron el apoyo de muchos docentes, padres y otros críticos de los sistemas educativos conservadores. Los gobiernos respondieron a esas demandas con la promoción de transformaciones en las estructuras educativas, que por su porte no podían ser gestionadas con las herramientas del planeamiento tradicional. Es así que se propiciaron nuevos tipos de planificación educativa, que implicaban cambios en sus concepciones y herramientas (COOMBS, 1970, p. 24).

En las naciones subdesarrolladas las tensiones experimentadas en las décadas de $1950 \mathrm{y}$ 1960 entre los sistemas educativos y su entorno social eran distintas a las de los países más ricos, pero no menos graves. Los problemas incluían inequidades en el gasto a la interna del sistema educativo, una creciente demanda de plazas como consecuencia del crecimiento demográfico, incremento de costos, cuellos de botella no financieros y escasez de oportunidades laborales para los más educados. A pesar de la expansión de la oferta educativa, su inadecuación para satisfacer las crecientes necesidades sociales era evidente, pero los recursos financieros disponibles

${ }^{1}$ Seguimos aquí a Coombs (1968 y 1970) y Varghese (2011). 
resultaban insuficientes para procesar el conjunto de las transformaciones necesarias. Naturalmente, esta restricción inducía a planear cuidadosamente las intervenciones en este sector (COOMBS, 1970, p. 26-31).

Pero más allá de esas notables diferencias entre los desafíos que enfrentaban en la década de 1960 los sistemas educativos de los países centrales y subdesarrollados, en esa coyuntura de cambios profundos los responsables del planeamiento educativo debían atender dos problemas principales, que signaron la evolución de la planificación como instrumento de políticas.

El primero fue el problema de la asignación de recursos, esto es, cómo orientar su distribución para satisfacer objetivos múltiples. Y esta tarea involucraba dos dimensiones: una, intersectorial, referida a la competencia de recursos con otras prestaciones sociales no educativas; otra, intrasectorial, relativa a las demandas de los distintos niveles del sistema de enseñanza.

El segundo problema fue el de la eficiencia: cómo maximizar los resultados de las inversiones educativas. Responder a este requerimiento implica contemplar tanto la eficiencia en la asignación, entendida como la obtención de retornos sociales al menos iguales a los de otras alternativas de inversión; y la eficiencia interna, que se define comparando resultados de calidad similar de una modalidad de intervención en relación a otras alternativas, dado un determinado nivel de inversión.

Como bien apunta Varghese (2011, p. 92), la respuesta dada a ambos problemas no ha sido una cuestión solamente técnica, sino también política: “...mientras la optimización es una preocupación mayor para los economistas y tecnócratas, la priorización es una preocupación más importante para el proceso de decisión política. Las decisiones sobre asignación de recursos a y entre unidades educativas refleja un trade-off entre lo que es óptimo, según lo postulan los planificadores, y lo que es deseable, según lo define el proceso político". ${ }^{2}$

Estos últimos apuntes nos conducen a preguntarnos: ¿qué características debería ostentar una "buena" planificación? ¿Qué variables se requieren tener en cuenta para que esta actividad técnico - intelectual pueda orientar e incidir positivamente en el desarrollo educativo? Desarrollamos el punto a continuación.

\section{Los desafíos para una planificación educativa efectiva}

Además de los análisis de base económica para orientar el planeamiento y la evaluación de las políticas educativas, existen en la literatura especializada otras perspectivas que proponen ponderar su eficacia y la eficiencia con parámetros más amplios. Todos subrayan que la planificación y la decisión en el campo específico de la educación comparten algunas reglas básicas que son aplicables al diseño de cualquier política, pero que además de estas existen particularidades de este campo que es menester asumir.

Así, se ha enfatizado la complejidad intrínseca de los problemas educativos, que requieren por tanto abordajes alternativos para la planificación e implementación. También se han señalado algunas condiciones de éxito para las políticas educativas, extraídas de la experiencia. Y se ha puesto de relieve la necesidad de una adecuada articulación intra e intersectorial para favorecer los resultados de las políticas. Como se verá, de cada una de estas perspectivas se extraen enseñanzas para orientar una planificación educativa eficaz. Abordaremos cada uno de estos tópicos a continuación.

\footnotetext{
${ }^{2}$ La traducción es nuestra.
} 
Entender a las políticas educativas como problemas complejos

En las últimas décadas se han propuesto enfoques que pretenden ir más allá de una comprensión lineal del cambio en las políticas públicas. Inspirados en las ciencias naturales y en la ecología, tratan de capturar la complejidad de los problemas sociales, y desde ese entendimiento, propiciar formas de planificación de las intervenciones públicas más sofisticadas.

Este concepto de complejidad ha sido definido así:

[...] un objeto de estudio es complejo cuando es capaz de sorprender a un observador debido a que su comportamiento no puede ser reducido al comportamiento de sus partes. Los sistemas sociales complejos requieren formas de conocimiento complejas, fundamentalmente, formas de comprensión que sean sensibles al contexto, al tiempo, al cambio, a los eventos, creencias y deseos, al poder, a los bucles de retroalimentación, y a la circularidad (TSOUKAS, 2005, p. 4).

De la definición se extrae el rol determinante que desempeñan los contextos en los que cualquier política pretenda implementarse. Para dar cuenta de las distintas situaciones posibles, Snyder (2013) y Snowden y Boone (2007) han elaborado la siguiente tipología:

- Contextos simples. Están determinados por la causalidad lineal. Las situaciones pueden ser claramente definidas, y desde el inicio se pueden identificar las respuestas apropiadas. Por ello, el rol de los decisores es definir la política y encomendar su puesta en práctica a los funcionarios encargados, de acuerdo a procedimientos de trabajo estandarizados. Coincide con las modalidades tradicionales de implementación, conocidas como "top down" (de arriba hacia abajo).

- Contextos complicados. La relación causa - efecto no es evidente, y requiere ser investigada. El decisor requiere de altas dosis de asesoramiento experto y de análisis de datos para formular una hipótesis de trabajo. Recién luego de producida la información están dadas las condiciones para intervenir.

- Contextos complejos. Se trata de situaciones de gran labilidad e imprevisibilidad, donde múltiples variables se hallan en movimiento permanente. No sólo son inciertas las respuestas, sino que las mismas incógnitas son desconocidas. Ante este escenario, el responsable de las políticas se ve exigido a altos niveles de interacción con el conjunto de los actores del sistema, como modalidad de aprendizaje de los procesos en curso, y a un modo experimental de gestión que incluye el ensayo y error. Modalidades de implementación clásicas estarían condenadas al fracaso; en su lugar, se debe permitir el surgimiento de "emergentes" que orienten los cambios. Se corresponde con la modalidad de implementación que en la teoría de las políticas públicas se ha denominado como "bottom-up" (de abajo hacia arriba).

- Contextos caóticos. Alude a situaciones excepcionales, donde no resulta posible planificar acciones a mediano o largo plazo, sino que deben tomarse medidas para atender la emergencia. A diferencia del tipo anterior, aquí se demanda la imposición de alguna forma de orden, para recién luego intentar transformarlo en un contexto complejo y procurar identificar regularidades y -en última instancia- soluciones, y prevenir nuevas crisis.

Aguerrondo (2014) adapta esta clasificación a las reformas educativas. Siguiendo las prescripciones recién recomendadas para cada escenario, propone el tipo de decisiones y los procesos de actuación preferibles en el Cuadro que sigue. 
Cuadro 1 - Tipos de contexto y toma de decisiones

\begin{tabular}{|c|l|l|}
\hline Contexto & \multicolumn{1}{|c|}{ Tipo de decisiones } & \multicolumn{1}{c|}{ Proceso de toma de decisiones } \\
\hline Simple & Dominio de las "buenas prácticas" & Percibir (detectar) $\rightarrow$ categorizar $\rightarrow$ responder \\
\hline Complicado & Dominio de los expertos & Percibir (detecta) $\rightarrow$ analizar $\rightarrow$ responder \\
\hline Complejo & Dominio de lo emergente & Probar $\rightarrow$ percibir $($ detectar $) \rightarrow$ responder \\
\hline Caótico & Dominio de la respuesta rápida & Actuar $\rightarrow$ percibir $($ detectar $) \rightarrow$ responder \\
\hline
\end{tabular}

Fuente: Aguerrondo (2014).

Para esta autora, las reformas educativas se dan siempre en contextos complejos, por lo que deberían descartarse estrategias de intervención y planificación clásicas, que parten de una clara separación entre los espacios de decisión y los de implementación, y no capturan adecuadamente las condicionantes contextuales. Por ello concluye:

Planificar en este contexto, y con el marco de la complejidad, ayuda a pensar la gestión de la incertidumbre, ya que esta es una de las características del mundo en que vivimos. Gestionar la incertidumbre requiere conceptualizaciones que permitan aprehenderla, lo que implica que quien gestiona (planifica, toma decisiones) debe tener la capacidad de pasar de una conceptualización binaria del futuro (una determinada característica del futuro, es o no es), a una de múltiples determinaciones (AGUERRONDO, 2014, p. 574).

\section{Asumir las particularidades de las transformaciones educativas}

Tomando como principal referencia la experiencia de las reformas implementadas en España, pero con pretensión de validez más amplia, Pedró y Puig (1999) identifican dos conjuntos de particularidades de las políticas educativas, referidas al ciclo de políticas y a su traslación en transformaciones sociales. Según estos autores, las particularidades de las políticas educativas vinculadas al ciclo de las políticas son las siguientes:

- En la fase de agenda o identificación del problema: el origen de la intervención estatal en el sector suele provenir de un desfase entre las orientaciones y objetivos del sistema escolar y los requerimientos del sistema social, o bien de un juicio de ineficacia del sistema escolar, entendido como un desequilibrio entre lo que debería conseguir y lo que en realidad consigue.

- En las fases de análisis y decisión: las reformas educativas requieren de un período adecuado de experimentación y de prueba, especialmente si afectan al currículum, a los métodos y los contenidos de la enseñanza. A su vez, las políticas educativas de mayor enjundia no pueden resolverse por vía administrativa y ni siquiera legal, sino que siempre es necesaria una negociación tendiente a conseguir los apoyos de los diferentes actores del sistema escolar. Estas peculiaridades le confieren a las políticas educativas una cierta parsimonia que las distingue de otras políticas.

- En la fase de implementación, las políticas educativas más importantesrequieren de una aplicación progresiva y gradual previa a su definitiva generalización. Este gradualismo es indicado para favorecer las adaptaciones a los contextos locales, para resolver sucesivamente los conflictos particulares que puedan ir surgiendo, y para posibilitar el financiamiento de las transformaciones más ambiciosas. "Así, si se há reformado la educación primaria y esta dura ahora seis años, habrá que esperar este período de tiempo 
como mínimo para poder decir que se há generalizado y completado la reforma" (PEDRÓ; PUIG, 1999, p. 57).

- En la etapa de evaluación, las políticas educativas demandan un monitoreo a lo largo de las diferentes fases, y no sólo en el momento de su culminación. Comprenden dos tipos de ponderaciones: el análisis longitudinal, aplicado a una cohorte de estudiantes a través del tiempo, y el análisis transversal, que enfoca distintas unidades o regiones en el mismo momento, con el propósito de determinar si hay cambios significativos en las sucesivas cohortes o si se producen disparidades regionales.

De acuerdo con los autores de referencia, las políticas educativas también reconocen especificidades en sus efectos:

- Sus objetivos suelen ser definidos más bien cualitativamente (equidad, calidad, democratización), y por lo tanto es difícil medir el éxito de una política.

- Los efectos se producen diferidos en el tiempo, por lo que debe aguardarse el cumplimiento integral de los ciclos de reforma para proceder a su evaluación final. Asimismo, el solapamiento con las políticas preexistentes (por ejemplo, viejos y nuevos planes de estudio) dificulta la ponderación de los resultados de unas y otras.

- Relación no lineal entre recursos asignados y resultados obtenidos, con un rango de variación mayor aún al esperable en otro tipo de políticas.

Por su parte, el psicopedagogo sueco Torsten Husén - inspirador de la reforma educativa instaurada en su país en la segunda posguerra-, propuso a partir de su propia experiencia seis lecciones para favorecer el éxito de las innovaciones educativas:

- Una reforma educativa solo tiene sentido si se acompaña una reforma social.

- Las reformas del sistema requieren de un correlato adecuado a escala local y en las mismas aulas.

- Vencer inercias requiere tiempo y gradualidad. En términos de los ya citados Pedró y Puig (1999, p. 63), "si hay un secreto en la planificación de las reformas, en la lentitud".

- La promoción de reformas ambiciosas necesita de consensos amplios, tanto por razones de legitimidad, como de eficacia: implementación efectiva y pervivencia en el tiempo.

- Las transformaciones suelen generar resistencias, que deben superar a través de una amplia información sobre los impactos de las nuevas políticas no sólo en el nivel del sistema, sino también de diferente colectivos e individuos.

- Las transformaciones educativas requieren de mecanismos aptos de monitoreo y evaluación de su desarrollo (HUSÉN, 1988).

- Favorecer la coordinación intra e intersectorial de las políticas educativas.

Si es cierto que las políticas educativas se desarrollan por definición en contextos complejos, y que la producción de innovación debe tomar en cuenta ciertas regularidades que le son propias, también es menester reconocer que de por sí resultan impotentes para obtener resultados ambiciosos si no se las articula con otras políticas públicas. 
Surge así el tópico de la coordinación, válido para todas las políticas, pero de especial importancia para la educación. Esto es así porque las políticas educativas ostentan dos características en un grado superlativo: a) pasivamente, son especialmente sensibles -receptivas- a la incidencia de otras políticas (económicas, sociales, etc.); y a su vez, en un sentido contrario, b) sus resultados tienen un alto impacto -repercusión- en otras esferas políticas, sociales y económicas (en la cultura ciudadana, en el mercado de trabajo, en el desarrollo económico, por citar sólo algunas). Esta tendencia favorable a la coordinación de políticas se ha reforzado en las últimas dos décadas por el prestigio de los enfoques multidimensionales del desarrollo, que superan los enfoques economicistas tradicionales incluyendo variables de desarrollo humano y social, entre las que se incluyen la salud, el género, las políticas, el ambiente, y por supuesto, la educación (SEN, 2000; NUSSBAUM, 2006).

De allí que coordinar las políticas educativas dentro de los distintos espacios del sistema educativo, pero también con otras políticas públicas, aparezca como indispensable. Y naturalmente, la planificación educativa es un espacio privilegiado para impulsar ese encastre. Abordando la función de la planificación en la coordinación de las políticas se ha sostenido:

[...] desde la óptica de la planificación, la coordinación supone algo más: de lo que se trata es de coordinar las políticas de manera que se logre integrar las distintas decisiones y acciones de gobierno dentro de una sola visión de conjunto y con una clara perspectiva de largo plazo. En otras palabras, se trata de coordinar con un sentido estratégico, se trata de coordinar para avanzar en determinada dirección; para promover determinados resultados de la acción pública en su conjunto, y no sólo de cada una de sus parcelas sectoriales o institucionales" (GARNIER, 2000, p. 15). ${ }^{3}$

Uno de los principales escollos para lograr esa coordinación son los diseños organizativos de las administraciones públicas -y en su seno, de los respectivos sistemas de planeamiento-, que no están orientados por macroobjetivos del Estado, sino que se especializan en el tratamiento de recortes parciales de la problemática social. En palabras de Ander Egg (1991, p. 33-34), "un sistema de planificación consiste, en esencia, en 'planificar la planificación', lo que implica ante todo una efectiva coordinación y comunicación entre los responsables de llevar a cabo los programas y los responsables de formularlos [...] tarea harto difícil a poco que se intente modificar el feudalismo de cada área de la administración pública y aún de cada departamento". Por tanto, el diseño institucional de los sistemas de planeamiento es un elemento clave para favorecer políticas educativas mejor coordinadas y, en última instancia, más eficaces.

Siguiendo a Persaud (2016), podemos dar cuenta de la evidencia acumulada en los últimos años, que indica que los enfoques multisectoriales de investigación, planeamiento e implementación para abordar los problemas educativos están adquiriendo un predicamento creciente en los organismos de la Naciones Unidas. Algunos de los temas clave mencionados en estos documentos de política son: alineación e integración de los planes sectoriales entre sí y dentro del contexto más amplio del desarrollo nacional y la reducción de la pobreza; creación de un entorno político favorable para la coordinación intersectorial; coordinación entre instituciones y agencias a todos los niveles para lograr objetivos de política interrelacionados; estructuras, procesos y mecanismos para facilitar la colaboración multisectorial; y apoyo a los acuerdos relativos al financiamiento y a los recursos humanos.

En esta línea, en un documento de la Secretaría General de las Naciones Unidas se aboga por enfoques holísticos e integradores para el desarrollo sustentable, comprendiendo una agenda

\footnotetext{
${ }^{3}$ La traducción es nuestra.
} 
articulada de soluciones económicas, ambientales y sociales, y por respuestas cohesivas e integradas a todos los objetivos vinculados para asegurar la viabilidad de transformaciones a gran escala. En relación a la implementación de políticas, el Informe subraya la necesidad concurrente de optimizar y coordinar los marcos de financiación e invertir en capacidades a nivel nacional integrando instituciones y recursos humanos, para incorporando los objetivos de desarrollo sostenible en los procesos de planificación nacional (UNITED NATIONS, 2015).

Por estas razones Persaud (2016) propone que la educación debería involucrarse en sistemas comprehensivos de planeamiento, que incluyeran dimensiones de integración verticales y horizontales con otras políticas y actores (ver Figura 1).

Figura 1 - Dimensiones del planeamiento integrado

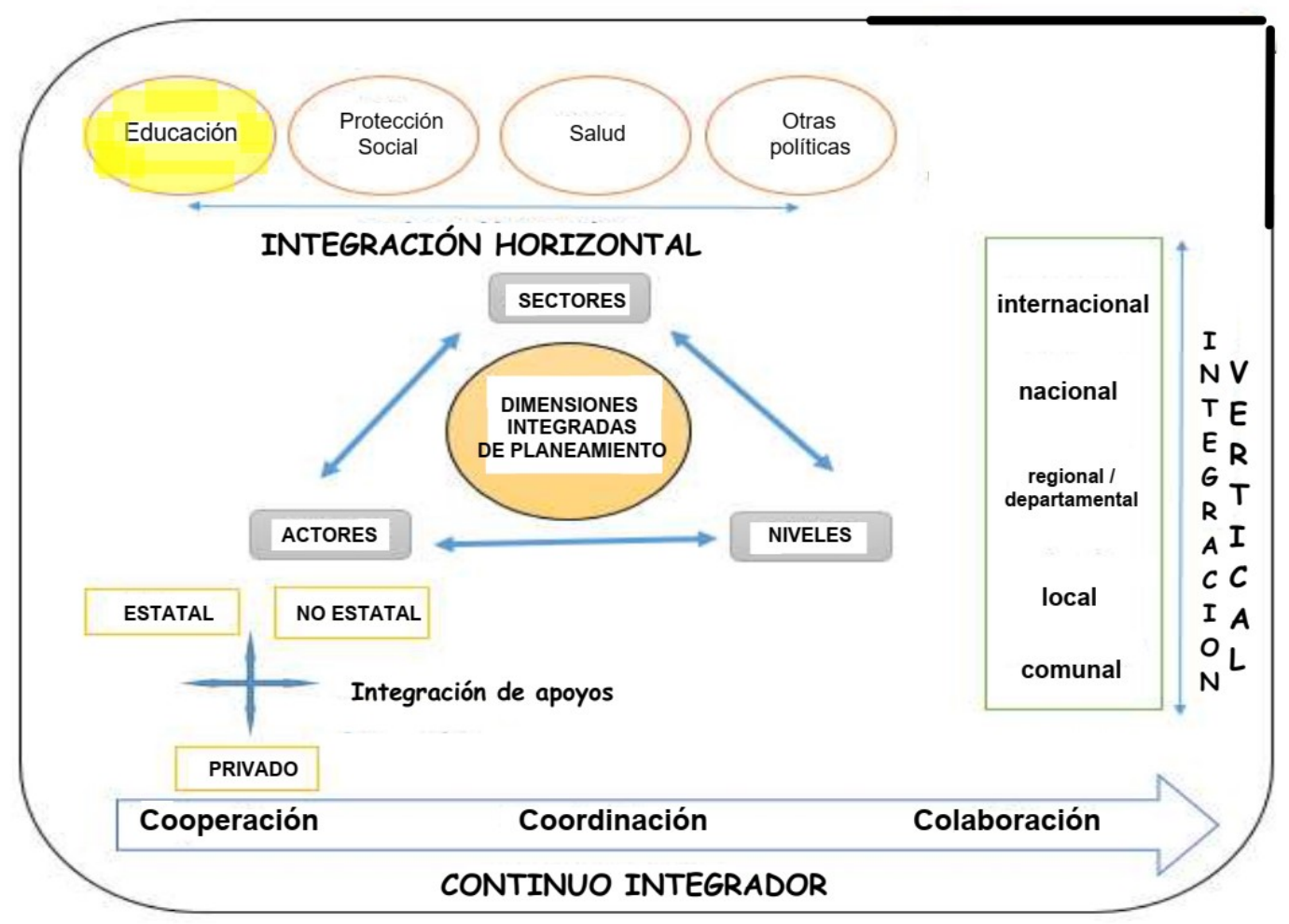

Fuente: Elaboración propia, en base a Persaud (2016, p. 13).

\section{Evolución del planeamiento educativo en América latina}

Hasta aquí hemos descrito en términos generales el surgimiento y evolución de las actividades de planificación educativa, y algunas variables relevantes a la hora de ponderar su adecuación y eficacia. Pero es necesario reparar con más detenimiento en las condiciones específicas que adquirió el fenómeno en nuestra región, que como se verá son por demás significativas.

La planificación estatal se afincó en América Latina a partir de los años cincuenta del siglo pasado, con fuerte impulso de la concepción desarrollista encarnada en la CEPAL, usina ideológica y técnica del movimiento. En la década siguiente, esta tendencia se trasladó al campo de la educación, también con fuerte protagonismo de instancias supranacionales. 
El Planeamiento Educativo: función política, modelos...

Fernández Lamarra y Aguerrondo (1977) identificaron un conjunto de factores explicativos de este trasiego:

- La extensión de la demanda por educación en todos los niveles.

- La creciente ampliación y complejización de la estructura académica y administrativa de los sistemas educativos.

- La necesidad de adecuar la educación a los imperativos del desarrollismo e integrarla a los planes económicos.

- La imitación de la planificación educativa desarrollada en otras regiones, especialmente en los países más desarrollados.

- La convicción de que el subdesarrollo se debía a la falta de organización y racionalización en procura de un objetivo.

- La prédica ideológica de los expertos en planificación, que la propugnaban como medio necesario para tramitar la modernización.

- La influencia de los organismos internacionales de promoción y financiamiento, que exigían el encuadre de todas las solicitudes de crédito en planes integrales.

Algunos eventos interamericanos de particular trascendencia jalonaron en las décadas de 1950 y 1960 el camino de la difusión y afianzamiento de la planificación educativa en la región. Entre los mismos se han destacado los siguientes:

- Conferencia de Ministros de Educación de América Latina celebrada en Lima (1956), en la que en base a la proto - experiencia desarrollada en Colombia se recomienda la aplicación de métodos de planificación educativa.

- Seminario Interamericano sobre Planeamiento Integral de la Educación de la OEA celebrado en Washington (1958), que dio lugar a cinco tomos en los que se asentó la base conceptual y metodológica para una planificación de tinte administrativo. En base a las recomendaciones ministeriales y a estos documentos la UNESCO y la OEA promovieron y financiaron la planificación educativa y, especialmente, la instalación de dependencias nacionales especializadas, más orientadas a la recolección de información y la realización de diagnósticos que a la formulación de planes.

- Conferencia Regional de Ministros de Educación y Ministros Encargados de la Planificación Económica realizada en Santiago de Chile (1962), orientada a articular las tareas de planificación económica con las del sector educativo. En cumplimiento de sus resoluciones, CEPAL y UNESCO crean una División de Planificación Educativa dentro del Instituto Latinoamericano de Planificación Económica y Social (ILPES), que formó técnicos de los distintos países.

- Conferencia Interamericana de Presidentes celebrada en Punta del Este (1962), que dio origen a la "Alianza para el Progreso". Puntualmente, entre sus recomendaciones figuró la de enmarcar todos los proyectos financiables externamente en planes de desarrollo. Este hecho propició la creación o fortalecimiento de dependencias nacionales especializadas (AGUERRONDO, 2007, p. 5-6; FERNÁNDEZ LAMARRA, 2006, p. 4748). 
Si se atiende a los énfasis conceptuales y finalistas que marcaron el proceso de instalación de la planificación educativa en la región, se identifica un primer sesgo hacia la satisfacción de la demanda ampliada por educación. A finales de la década de 1950, la universalización del acceso a la enseñanza primaria y -en menor medida- la respuesta a las mayores presiones de las clases medias en aras de la enseñanza media constituían las principales tareas de los planificadores, especialmente referida a los insumos necesarios para la evaluación de las soluciones adecuadas (recursos materiales, número de docentes, crecimiento demográfico, etc.). De acuerdo a la fecha definida para el cumplimiento de un objetivo, se estimaban en los planes los requerimientos de locales o maestros. Como demuestran algunos estudios posteriores, esas proyecciones fueron poco certeras, por la escasa calidad de los datos disponibles, pero fundamentalmente por su énfasis en la demanda social, y el menosprecio analítico de la voluntad política de los gobiernos para asegurar las inversiones necesarias.

Ya en la década de 1960, el foco de la planificación educativa en América Latina se desplaza desde la matrícula y el acceso, hacia los requerimientos del sistema productivo. Con una metodología puramente normativa ("planificación de recursos humanos"), se procuraba estimar las modalidades y duración de la enseñanza necesarias para satisfacer las demandas del crecimiento económico. Como era previsible, el cambiante entorno de las economías nacionales obstó al éxito de intentos de estas características.

No obstante, no en todos los países de la región la denominada "modernización educativa" de los años 50' y 60' adquirió las mismas características. Como se ha señalado en una investigación sobre el particular, "el modo en que los programas e iniciativas modernizadoras lograron (o no) articularse, institucionalizarse, crear tradiciones de investigación y de cooperación entre las agencias especializadas en la producción de datos y conocimientos, y las de gobierno y planificación, marcó un punto de diferenciación fuerte en la trayectoria posterior de los diferentes países" (PALAMIDESSI; GOROSTIAGA; SUASNÁBAR, 2014, p. 62).

Posteriormente, en la década del setenta se ensayó la Presupuestación y Planificación por Programa (PPB), de gran predicamento en Estados Unidos y extendida a diversos sectores de la actividad gubernamental. Básicamente, supuso una nueva negación de los elementos contextuales que impedían el suceso de los planes educativos, procurando soslayar ese análisis mediante un conocimiento técnicamente más ajustado del objeto de la intervención, mayor preocupación por la eficiencia del gasto y la realización de evaluaciones periódicas (MC GINN; PORTER, 2005).

El desencanto con los sucesivos intentos reseñados condujo, sobre finales de la década de 1970, a la crisis de los paradigmas y de las prácticas imperantes en la planificación educativa en nuestra región. Más allá de ciertos avances en términos de información estadística disponible, la planificación no había logrado constituirse en una herramienta válida y confiable para el desarrollo de las políticas educativas.

Fernández Lamarra (2006, p. 50-51) entiende que las principales limitaciones de las experiencias que condujeron a dicha crisis fueron las siguientes:

- utilización de un modelo conceptual-metodológico de carácter esencialmente normativo;

- desarticulación del planeamiento con las otras dimensiones (política y administrativa) que componen el proceso de gobierno;

- planes de educación que eran esencialmente diagnósticos, y que incluían objetivos y metas muy ambiciosas sin reparar en las realidades nacionales, ni analizar su viabilidad; 
- desarticulación de las oficinas de planeamiento con los otros sectores ministeriales y, muchas veces, con las actividades educativas;

- escasa o nula participación de los destinatarios de los planes (funcionarios, técnicos, docentes, comunidad, etc.) en el proceso de elaboración de los mismos;

- muy escasa investigación e innovación para generar los conocimientos requeridos para la formulación de los planes;

- tratamiento muy global (de macro planificación) tanto en los diagnósticos, en las metas y en los objetivos como en los programas, sin atender a realidades muy diversas, diferenciadas y específicas en cada una de las regiones de cada país;

- escaso énfasis en los aspectos cualitativos de la educación, ya que los planes y trabajos desarrollados tenían un marcado énfasis cuantitativo.

Como puede apreciarse, las estrategias promovidas en los años sesenta y setenta ignoraban los principales resguardos para propiciar un planeamiento eficaz a los que hicimos referencia en el apartado anterior. No asumían la complejidad de los problemas educativos, desdeñaban las particularidades del ciclo de construcción de las políticas educativas y soslayaban la articulación con otras políticas y programas.

A partir de los años ochenta, se cuestiona también la capacidad y oportunidad de la planificación como instrumento apto para promover el desarrollo de los países de la región. En un contexto de restricción de fines, funciones y aparatos estatales las manifestaciones más ambiciosas de planeamiento aparecían como elementos claramente disfuncionales para la constitución de estados más pequeños, menos costosos, y reducidos en sus cometidos a la fijación de reglas de juego para la actuación de los protagonistas privados. Se trasladó así al campo de la educación el cuestionamiento a los estados de bienestar expandidos, al que hiciéramos referencia más arriba en este texto.

Como consecuencia de esta doble impugnación, los modelos más pretenciosos de planificación de las décadas anteriores fueron sustituidos durante los años 80s. y 90s. por la promoción de programas y proyectos sectoriales más acotados, por lo general financiados con fondos de instituciones financieras internacionales y objetivos mucho más específicos. El énfasis se trasladó desde la planificación al "management" de la educación. Y si bien los discursos frecuentemente reivindicaban la función de los departamentos de planeamiento, es la práctica fueron reducidos al rol de unidades operativas para la ejecución de tareas rutinarias (MATUS, 2007; POGGI, 2011). En lugar de prohijar el surgimiento y consolidación de burocracias de carrera especializadas en el seno de los ministerios de educación, estos proyectos por lo general acudieron a la contratación a término de especialistas en distintas áreas vinculadas al objeto educativo.

\section{Los modelos de planificación}

Los limitados logros de algunas de las experiencias de planificación que han sido reseñadas hasta aquí indujeron a la creación de esquemas alternativos para articular objetivos de políticas y gestión pública. Es así que comenzaron a perfilarse diferentes modelos de planificación, rubro en el cual estudios y experiencias producidos en nuestra región han realizado aportes muy significativos. 
En la literatura especializada se distinguen dos modelos básicos de planificación: el normativo - al que podría también identificarse como el "modelo clásico"- y su alternativa, el modelo de planificación estratégica. Un autor ineludible en este tema, el chileno Carlos Matus (1972, p. 101), reseñaba ya a comienzos de la década de 1970 los caracteres del modelo clásico:

El procedimiento normativo define un curso del proceso de desarrollo que comprende las acciones 'necesarias' para cumplir determinados objetivos fijados 'a priori', pero sin utilizar hasta sus últimas consecuencias el conocimiento de los factores que explican y determinan la conducta; las acciones propuestas no surgen de las funciones reales de comportamiento, sino que se sobrepone a éstas una norma de conducta coherente con los objetivos. Entre la situación inicial y el objetivo hay una trayectoria eficaz que 'debe' sustituir al comportamiento real; esa trayectoria eficaz es simplemente un requisito de la norma-objetivo. El modelo no surge dialécticamente de la realidad, sino que se deduce de la norma-objetivo. Modelo y realidad están en planos diferentes, sin posibilidad cierta de contacto, separándolos la misma diferencia que existe entre comportamiento regla y regla ideal, entre necesidad y posibilidad. estratégico:

El mismo autor presentaba entonces las diferencias introducidas por el planeamiento

El procedimiento estratégico, en cambio, supone una respuesta del sistema ante alteraciones deliberadas, respuesta que puede orientarse hacia el cumplimiento de objetivos conscientemente elegidos. La norma es aquí el punto 'hacia' el cual se pretende encauzar el comportamiento del sistema. La trayectoria del proceso para alcanzar la norma no deriva de ésta, sino de ajustes posibles y sucesivos en el comportamiento del sistema. El procedimiento estratégico, lejos de superponerse a la realidad, emerge de ella, es la misma realidad y a la vez se distingue de ésta porque busca un medio de modificarla basándose en un conocimiento preciso de su funcionamiento (MATUS, 1972, p. 101).

Como puede deducirse de las citas anteriores, el modelo normativo de planificación es tributario de una concepción positivista, sustentada en tres supuestos básicos:

i. la posibilidad de anticipar los problemas más importantes a enfrentar en el futuro;

ii. el conocimiento actual de los planificadores sobre las formas más efectivas para la solución de esos problemas; y

iii. la aplicación de los medios específicos para la solución de los problemas no representa -en sí misma- un problema, sea por la no asunción de esta responsabilidad por parte de quien elabora el plan, o por la confianza en que quien decidirá e implementará la política cuenta con el poder y la competencia necesaria para imponer las recomendaciones (MC GINN; PORTER, 2005).

El modelo clásico constituye, por tanto, una expresión manifiestamente optimista en la capacidad moldeadora de la realidad que ostentaría el conocimiento especializado, que subestima la complejidad intrínseca de los procesos sociales y más específicamente, su racionalidad política.

La planificación estratégica, por su parte, se ha convertido en un pilar central de la modernización del sector público. De forma cada vez más persistente, las organizaciones públicas desarrollan sistemas de planificación, ya sea generales (planificaciones estratégicas o planes operativos anules), o parciales (planes financieros, de procesos o acciones específicas de áreas). 
El foco en la planificación se asocia con la búsqueda de una gestión estratégica de la organización enfocada en promover una distribución funcional eficiente basada en objetivos comunes, y vincular la gestión interna de la organización para alcanzar resultados que creen valor público.

Una de las definiciones más usadas del concepto de planificación estratégica refiere a: "un esfuerzo deliberado y sistemático para producir decisiones y acciones fundamentales que dan forma y guían qué es una organización, qué hace y por qué” (BRYSON, 2004, p. 38). ${ }^{4}$

Aunque puede entenderse como una definición genérica, Bryson señala que la planificación estratégica no debe verse como un proceso específico o preciso que debe seguirse en cada ocasión; más bien, es un "conjunto de conceptos, procedimientos y herramientas", que se debe usar sólo si ayuda a pensar y actuar estratégicamente. Por lo tanto, la planificación no es un fin en sí mismo, sino un método para alcanzar resultados deseables. Así, la planificación busca incorporar un enfoque más amplio para la gestión de las organizaciones y sus tareas (POISTER; STREIB, 1999).

Teniendo en cuenta estos aspectos: "la planificación estratégica no promete que las decisiones serán correctas; solo que se harán con previsión” (JOHNSTON, 2002, p. 30). En ese sentido, es importante enfatizar que la planificación no es un proceso fijo ni aislado; más bien es un proceso insertado en el entorno y sujeto a las características de la organización en la que tiene lugar. Por lo tanto, el proceso en el que se desarrolla la planificación no es estable, ni fácilmente predecible o las consecuencias pueden ser predeterminadas. Una planificación efectiva depende de la capacidad de predecir de la manera más precisa posible cómo cambiará el entorno durante la implementación de la política (MINTZBERG, 1994). sigue.

Las diferencias entre los dos modelos de planificación se esquematizan en el cuadro que

Cuadro 2 - Planificación normativa y estratégica: comparación

\begin{tabular}{|c|c|}
\hline Normativa & Estratégica \\
\hline estabilidad y certidumbre & inestabilidad y turbulencia \\
\hline armonía de interesses & concertación de intereses contrapuestos \\
\hline neutralidad respecto al objeto & compromiso con el objeto \\
\hline Regulación & arbitraje \\
\hline Equilíbrio & compensación de desigualdades \\
\hline visión lineal del conocimiento y la acción & visión circular y recurrente \\
\hline predicción sobre el futuro (anticipado) & previsión (anticipar condiciones de viabilidad) \\
\hline cálculo unidireccional (un solo planificador) & cálculo interactivo (todos los actores planifican) \\
\hline solución única y homogénea & soluciones diversas y selectivas \\
\hline separación del sujeto / objeto de la planificación & integración del sujeto y objeto \\
& de la planificación \\
\hline El planificador decide qué y cómo hacerse & el planificador debe crear las condiciones para que el \\
\end{tabular}

Fuente: Fernández Lamarra (2006, p. 54).

\footnotetext{
${ }^{4}$ La traducción es nuestra.
} 
Anudando estos conceptos con los desarrollos de los apartados anteriores de este capítulo, el concepto de "estrategia" conlleva la asunción de las dos dimensiones de viabilidad de cualquier proyecto comprehensivo de transformaciones. Por un lado, una viabilidad técnica o económica, anclada en la relación costo - beneficio de las medidas a implementar. Por otro - y esta es la novedad principal- la eficacia política, entendida como la capacidad de un conjunto de proyectos para cambiar la estructura de fuerzas sociales. La estrategia es, así, tanto un proyecto político en tanto implica definir la dirección y las formas de encausar la realidad hacia un rumbo específico, como una actitud y un método, que demanda la integración coherente de las partes restantes de la planificación con dichas definiciones. Es posible, por ello, que en ciertos momentos la viabilidad política difiera de la trayectoria (secuencia) técnica, y en tales casos ésta última deberá ajustarse a la primera. Asimismo, el patrón de desarrollo de un plan, entendido como el ajuste de cada uno de sus proyectos a la totalidad, puede variar según se modifiquen las situaciones materiales que constituyen su referencia.

Por este carácter social y político de la planificación es que Matus (1972, p. 118-119) asigna vital importancia al proceso intelectual de formación de conciencia, y al proceso material de creación de condiciones para el cambio. El primero es una tarea abstracta, por medio de de la cual los grupos sociales integran sus situaciones de partida en una visión de conjunto que las explica como parte de un proceso con un sentido determinado, en una secuencia originariamente de carácter intelectual y posteriormente social, que le otorga legitimidad y vivencia real. En cambio, el proceso material de creación de condiciones para el cambio es una acción concreta, que modifica progresivamente la posición de los grupos sociales frente a los cambios, alterando la posición relativa de cada uno de ellos ante las nuevas situaciones que el plan va delineando. Ambos procesos -el intelectual y el material- se influyen recíprocamente, por cuanto el primero abre nuevas posibilidades de acciones materiales, y éstas crean otras condiciones para la formación de conciencia.

De la misma forma en que la planificación normativa se sustentaba en la racionalidad positivista, los desarrollos anteriores asumieron progresivamente un enfoque dialéctico. Surgió así una variante de cuño latinoamericano de la planificación estratégica, a la que se la denominó "planificación estratégica situacional" o directamente "planificación situacional". En el cuadro siguiente se comparan supuestos fundamentales del modelo clásico y de esta expresión renovadora.

Cuadro 3 - Comparación entre planificación normativa y situacional

\begin{tabular}{|l|c|c|}
\hline & Planificación normativa & Planificación situacional \\
\hline 1. Objetivos & normativos & políticos \\
\hline 2. Agentes & técnicos & $\begin{array}{c}\text { políticos/técnicos/ } \\
\text { administrativos }\end{array}$ \\
\hline 3. Oponentes & no se consideran & se tienen en cuenta \\
\hline 4. Medios & medios asignados & creación de medios \\
\hline 5. Tipo de cálculo & no se considera & estratégico y táctico \\
\hline 6. Restricciones & materiales & políticas \\
\hline 7. Ámbito de la planificación & todo el sistema & dominio situacional específico \\
\hline
\end{tabular}

Fuente: Aguerrondo (2007, p. 10), adaptación de Matus (1987). 
En esta expresión del proceso de planificación, entonces, se sustituye la concepción evolutiva lineal del modelo clásico por una secuencia de etapas sucesivas, que inciden una sobre otra, según las condicionantes creadas por un entorno social incierto y cambiante, pautado por la existencia de actores con perspectivas e intereses divergentes. La racionalidad técnica se redefine, entonces, como un instrumento al servicio de la estrategia política definida para acompañar ese proceso.

\section{Reflexiones finales: planeamiento y políticas educativas en América Latina, hoy}

Del recorrido conceptual ofrecido debería emerger con claridad que las actividades de planeamiento de la educación pueden representar bastante más que un operativo meramente técnico de recolección de información estadística y articulación de objetivos, metas e instrumentos de políticas. En sus expresiones más virtuosas, constituyen una estrategia de elaboración de políticas que se ofrece como alternativa a la consabida dicotomía entre políticas "racionales" y apoyadas en expedientes técnicos, por un lado, y otras principalmente anidadas en consensos políticos y sociales coyunturales, por otro.

Más allá del interés académico o profesional por los ensayos de planificación de esa naturaleza, en la actualidad la reivindicación de su función político - institucional es especialmente relevante para la discusión sobre las reformas pendientes en los sistemas de educación de los países de América Latina.

Esto es así porque aparentemente ha culminado un ciclo de producción de políticas, iniciado en la primera década de este siglo, en el que se procuraron consensos amplios y arquitecturas participativas para el diseño de las políticas educativas, principalmente encarnadas en leyes y planes nacionales de educación (BENTANCUR, 2008; LÓPEZ, 2007; SAFORCADA; VASSILIADES, 2011; SUASNÁBAR, 2017). Sus limitados resultados y -más aún- los enormes desafíos de resolución aún pendiente para nuestros sistemas, han producido una extendida reacción de escepticismo sobre la procedencia de ese camino.

Ese agotamiento en la búsqueda de consensos para las transformaciones, que por su misma vocación inclusiva quedó frecuentemente atrapado en una composición de visiones e intereses de múltiples actores más preocupada por la coyuntura que por las construcciones de mediano y largo plazo, propicia hoy el ensayo de otras estrategias "eficaces", "sustentadas técnicamente", que puedan liberarse de los "obstáculos de la política" y "hacer lo que se debe" para solucionar los problemas de la enseñanza en el corto plazo.

Si algo debemos aprender de nuestra experiencia en el planeamiento de la educación, es que este modelo normativo ya ha sido aplicado en las últimas décadas del siglo anterior en muchos de nuestros países, sin haber resultado capaz de resolver esos mismos problemas. Esa modalidad tradicional de construcción de las políticas pudo adjudicarse algunos logros inmediatos, pero al carecer de un sustento político y social suficiente, resultó impotente para prohijar reformas sostenidas en el tiempo, imprescindibles para cualquier transformación educativa de gran calado.

Es menester, entonces, recuperar la concepción de la educación como un problema complejo, de improbable resolución a través de visiones unidimensionales y expedientes meramente instrumentales. Nuevos compromisos entre saberes técnicos y gestión política de las reformas son necesarios. Y los modelos más maduros de planeamiento educativo aparecen como una estrategia válida para alcanzar ese objetivo. 


\section{Referencias}

AGUERRONDO, I. Planificación educativa y complejidad: gestión de las reformas educativas. Cadernos de Pesquisa, São Paulo, v. 44, n. 153, p. 548-578, jul./set. 2014. DOI: http://dx.doi.org/10.1590/198053142910

AGUERRONDO, I. Racionalidades subyacentes en los modelos de planificación (educativa). Buenos Aires: IIPE-UNESCO, 2007.

AGUILAR VILLANUEVA, L. Estudio introductorio. In: AGUILAR VILLANUEVA, L. (Comp.). El estudio de las políticas públicas. México: M. A. Porrúa, 1992. p. 15-74.

ANDER-EGG, E. Introducción a la planificación. Madrid: Siglo XXI, 1991.

BENTANCUR, N. La nueva agenda de las políticas educativas en el Cono Sur (Argentina, Chile y Uruguay 2005-2008). Revista Debates, Porto Alegre, v. 2, n. 2, p. 272-298, jul./dez. 2008. DOI: https://doi.org/10.22456/1982-5269.3066

BRYSON, J. Strategic management and planning. In: PETERS, G.; PIERRE, J. (Eds.). Handbook of public administration. London: Sage, 2004. p. 38-47.

COOMBS, P. H. What is educational planning? Fundamentals of Educational Planning, n. 1. Paris: IIEP-UNESCO, 1970.

COOMBS, P. H. The world educational crisis: a systems analysis. New York: Oxford University Press, 1968.

FERNÁNDEZ LAMARRA, N. Reflexiones sobre la planificación de la educación en la Argentina y en América Latina: evolución, crisis, desafíos y perspectivas. In: FERNÁNDEZ LAMARRA, N. (Comp.). Política, planeamiento y gestión de la educación: modelos de simulación en Argentina. Buenos Aires: EDUNTREF, 2006. p. 45-65.

FERNÁNDEZ LAMARRA, N.; AGUERRONDO, I. La planificación educativa en América Latina: una reflexión a partir de la opinión de los planificadores de la región. Buenos Aires: CEPAL, 1977.

GARNIER, L. Función de coordinación de planes y políticas. Santiago de Chile: ILPES CEPAL, 2000. (Serie Gestión Pública, 1)

HADDAD, W. D.; DEMSKY, T. Education policy-planning process: an applied framework. Fundamentals of Educational Planning, n. 51. París: ERIC, 1995.

HUSÉN, T. Nuevo análisis de la sociedad del aprendizaje. Barcelona: Paidós, 1988.

JOHNSTON, J. Strategic management practice and participation in the public sector: illusion or rational instrumentality in pluralist approaches to employment relations? Employment Relations Records, v. 2, n. 1, p. 27-40, 2002.

LÓPEZ, N. Las nuevas leyes de educación en América Latina: una lectura a la luz del panorama social de la región. Buenos Aires: IIPE-Unesco, 2007.

MARSHALL, T. H. Class, citizenship, and social development. New York: Doubleday, 1965.

MATUS, C. Estrategia y plan. México DF: Siglo XXI Editores, 1972. 
MATUS, C. Planificación y gobierno. Revista de la Cepal, n. 31, p. 161-178, feb. 1987. DOI: https://doi.org/10.18356/e00423c2-es

MATUS, C. Los tres cinturones del gobierno. San Justo: UNLM-CIGOB-ALTADIR, 2007.

MC GINN, N.; PORTER, G. L. El supuesto fracaso de la planificación educativa en América Latina. Revista Latinoamericana de Estudios Educativos, Ciudad do México, v. 35, n. 3-4, p. 77-113, 2005.

MINTZBERG, $\mathrm{H}$. The rise and fall of strategic planning. Hemel Hempstead: Prentice Hall, 1994.

MISHRA, R. El Estado de Bienestar después de la crisis: los años ochenta y más allá. IN: MUÑOZ DEL BUSTILLO, R. (Comp.). Crisis y futuro del Estado de Bienestar. Madrid: Alianza Universidad, 1989. p. 55-79.

NUSSBAUM, M. C. Education and democratic citizenship: capabilities and quality education. Journal of Human Development, v. 7, n. 3, p. 385-395, nov. 2006. DOI: https://doi.org/10.1080/14649880600815974

PALAMIDESSI, M.; GOROSTIAGA, J.; SUASNÁBAR, C. El desarrollo de la investigación educativa y sus vinculaciones con el gobierno de la educación en América Latina. Perfiles Educativos, v. 36, n. 143, p. 49-66, ene. 2014. DOI: https://doi.org/10.1016/s0185$\underline{2698(14) 70609-9}$

PEDRÓ, F.; PUIG, I. Las reformas educativas: una perspectiva política y comparada. Barcelona: Paidós, 1999.

PERSAUD, A. Integrated planning for education and development. Background paper prepared for the 2016 Global Education Monitoring Report. París: UNESCO, 2016.

POGGI, M. Key issues in educational agendas: new perspectives for planning in Latin America. In: BRAY, M.; VARGHESE, N. V. (Eds.). Directions in educational planning: international experiences and perspectives. París: IIEP-UNESCO, 2011. p. 241-252.

POISTER, T.; STREIB, G. Strategic management in the public sector: models, concepts, and processes. Public Productivity \& Management Review, v. 22, n. 3, p. 308-325, mar. 1999. DOI: $\underline{\text { https://doi.org/10.2307/3380706 }}$

RUSSELL, B. Autoridad e individuo. México DF: Fondo de Cultura Económica, 1995.

SAFORCADA, F.; VASSILIADES, A. Las leyes de educación en los comienzos del siglo XXI: del neoliberalismo al postconsenso de Washington en América del Sur. Educação \& Sociedade, Campinas, v. 32, n. 115, p. 287-304, abril-jun. 2011. DOI: https://doi.org/10.1590/s0101$\underline{73302011000200003}$

SNOWDEN, D. J.; BOONE, M. E. A leader's framework for decision-making. Harvard Business Review, v. 85, n. 11, p. 68-76, nov. 2007.

SEN, A. Desarrollo y libertad. Barcelona: Planeta, 2000.

SNYDER, S. The simple, the complicated, and the complex: educational reform through the lens of complexity theory. OECD Education Working Papers, n. 96, dec. 2013. DOI: https://doi.org/10.1787/5k3txnpt1lnr-en 
SUASNÁBAR, C. Los ciclos de reforma educativa en América Latina: 1960, 1990 y 2000. Revista Española de Educación Comparada, Madrid, n. 30, p. 112-135, jul./dec. 2017. DOI: https://doi.org/10.5944/reec.30.2017.19872

TSOUKAS, H. Complex knowledge: studies in organizational epistemology. Oxford: Oxford University Press, 2005.

UNITED NATIONS. Policy integration in government in pursuit of the sustainable development goals. Report of the expert group meeting held on 28 and 29 January 2015 at United Nations Headquarters, New York (No. E/CN.16/2015/CRP.2). United Nations: Department of Economic \& Social Affairs, 2015.

VARGHESE, N. V. State is the problem and state is the solution: changing orientations in educational planning. In: BRAY, M.; VARGHESE, N. V. (Eds.). Directions in educational planning: international experiences and perspectives. París: IIEP-UNESCO, 2011. p. 89-108.

Recebido em: 16/09/2019

Versão corrigida recebida: 02/12/2019

Aceito em: 03/12/2019

Publicado online: 07/12/2019

\section{Nicolás Bentancur}

Profesor titular del Departamento de Ciencia Política, Facultad de Ciencias Sociales, Universidad de la República. Integrante del Sistema Nacional de Investigadores de Uruguay. 